\title{
Processing of spectrally resolved $x$-ray images of inertial confinement fusion implosion cores recorded with multimonochromatic x-ray imagers
}

\author{
T. Nagayama, ${ }^{1, a)}$ R. C. Mancini, ${ }^{1}$ R. Florido, ${ }^{1, b)}$ R. Tommasini, ${ }^{2}$ J. A. Koch, ${ }^{2}$ J. A. Delettrez, ${ }^{3}$ \\ S. P. Regan, ${ }^{3}$ and V. A. Smalyuk ${ }^{3, c)}$ \\ ${ }^{1}$ Department of Physics, University of Nevada, Reno, Nevada 89557, USA \\ ${ }^{2}$ Lawrence Livermore National Laboratory, Livermore, California 94550, USA \\ ${ }^{3}$ Laboratory for Laser Energetics, University of Rochester, New York 14623, USA
}

(Received 6 January 2011; accepted 17 March 2011; published online 2 May 2011)

\begin{abstract}
We discuss the processing of data recorded with multimonochromatic x-ray imagers (MMI) in inertial confinement fusion experiments. The MMI records hundreds of gated, spectrally resolved images that can be used to unravel the spatial structure of the implosion core. In particular, we present a new method to determine the centers in all the array of images, a better reconstruction technique of narrowband implosion core images, two algorithms to determine the shape and size of the implosion core volume based on reconstructed broadband images recorded along three-quasiorthogonal lines of sight, and the removal of artifacts from the space-integrated spectra. (c) 2011 American Institute of Physics. [doi:10.1063/1.3580513]
\end{abstract}

\section{INTRODUCTION}

Inertial confinement fusion (ICF) is a technique to achieve high gain energy production by compressing fuelfilled spherical capsules taking advantage of the capsule ablation driven directly or indirectly by laser beams. ${ }^{1-4}$ To maximize the energy gain, several schemes are under research such as central hot spot ignition, fast ignition, and shock ignition, each of which relies on different spatial structure of temperature and density achieved inside the core at the collapse of the implosion. ${ }^{3-6}$ The ideal implosion requires spherically symmetric compression, which is hard to achieve due to laser nonuniformity, laser power imbalance, and surface roughness amplified by various hydrodynamic instabilities. To study the symmetry and spatial structure of the compressed core, various imaging instruments have been developed including the gated monochromatic $\mathrm{x}$-ray imager, ${ }^{7,8}$ monochromatic $\mathrm{x}$-ray imaging with bent crystals, ${ }^{9-11}$ and multimonochromatic x-ray imager (MMI) applied to indirect-drive, ${ }^{12-15}$ and direct-drive ${ }^{16,17}$ laser-driven implosions. Monochromatic images (or narrowband images) show core images of specific line transitions from spectroscopic tracers, which have potential to unveil the spatial structure of temperature and density in the core.

The MMI instrument records a large number of gated, spectrally resolved implosion core images, each of which is formed by photons in a slightly different energy range. One advantage of the MMI instrument over other monochromatic imagers is that MMI data are rich in information and one can extract different types of data such as broadband and narrowband images, and space-integrated spectra by postprocessing

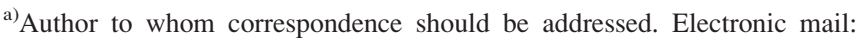
taisuke@unr.edu.

b)Present address: Departamento de Física, Universidad de Las Palmas de Gran Canaria, 35017 Las Palmas de Gran Canaria, Spain.

${ }^{c}$ Present address: Lawrence Livermore National Laboratory, Livermore, CA 94550.
}

the raw MMI data. Thus, a good postprocessing method is crucial to maximize the usefulness and diagnostic impact of the MMI data.

The MMI allows a few images within a fixed x-ray energy bandwidth to be summed up in order to produce an image characteristic of that bandwidth. Although this method has the advantage of increasing the signal-to-noise ratio of the resulting image, it has the drawback of giving up some of the spectral resolution of the instrument. ${ }^{12}$ Alternatively, the small change in the spectral range between adjacent images can be used to reconstruct a narrowband image from the addition of small portions of several original images. ${ }^{14,18}$ The first processing method of MMI data was developed by Welser et al. ${ }^{12}$ It handles each core subimage individually and reconstructs narrowband core images by averaging several core subimages aligned by their centers. There are several limitations in this first method. First, the alignment of selected core subimages is difficult due to the center determination based on individual subimages. Second, the reconstructed image has a slightly different narrowband range across the image. Third, this image reconstruction is based on working with whole core images from the MMI data. As one whole core image spans a certain photon energy range, this energy range becomes the narrowest bandwidth required for the image reconstruction, which is often broader than the desired energy range. These points were improved by Izumi et al. ${ }^{14}$ who used a fast Fourier transform (FFT) to analyze and find the centers of all the core subimages simultaneously. In turn, this procedure led to a better alignment of the subimages and allowed reconstruction of images within a narrower bandwidth.

This paper considers further advancements and new developments in the MMI data processing protocol. First, we discuss a new method to simultaneously extract all the centers of the implosion core images. This method is applied directly to real space, instead of Fourier space, thus resulting in an accurate and faster technique, while providing more 
direct control of the process. Based on the centers as reference points, one can then reconstruct broadband and/or narrowband images using the method published by Izumi et al. ${ }^{14}$ However, we point out a problem with this method and its correction based on an intensity-weighted reconstruction. Then, we look into two applications of the extracted broadband images. One is the creation of the MMI mask image of the implosion core and its application to the extraction of the space-integrated spectrum. Another is the determination of the shape and size of the implosion core volume. In Sec. II, we briefly describe the experimental setup used to record the MMI data. In Sec. III, we present a new center determination algorithm and its validity. In Sec. V, we review the image reconstruction algorithm and point out an intensity weighted reconstruction option to fix an artifact. In Sec. V, we show how to use the broadband image to improve the quality of the space-integrated spectrum. In Sec. VI, two algorithms to estimate the size and shape of the implosion core volume using MMI data simultaneously recorded along three lines of sight are introduced. Finally, Sec. VII summarizes all the developments and improvements, and discusses the significance of the new findings.

\section{EXPERIMENTS AND MMI DATA}

In a series of direct-drive ICF experiments performed at the OMEGA laser facility of the Laboratory for Laser Energetics at the University of Rochester, NY, 60 OMEGA laser beams were used to irradiate the surface of gas-filled plastic shell targets. The laser beams were frequency tripled so that the wavelength of the laser light irradiating the target was $1 / 3 \mu \mathrm{m}$, with a total laser energy on target of $19 \mathrm{~kJ}$ and a power imbalance characterized by a root-mean square of $3 \%$. In order to achieve the best irradiation uniformity conditions on target, the laser beams were smoothed with distributed phase plates, ${ }^{19}$ distributed polarization rotators, ${ }^{20}$ and smoothing by spectral dispersion. ${ }^{21}$ The laser pulse shape had a duration of approximately $2 \mathrm{~ns}$ and a shape designed to drive a low-adiabatic type of implosion (see Fig. 1). The shells had an interior radius $R=400 \mu \mathrm{m}$ and thickness $\Delta R=27 \mu \mathrm{m}$, and included an embedded thin Ti-doped tracer layer. The shells were filled with 20 atm of deuterium and $0.072 \mathrm{~atm}$ of argon. The tracer amount of Ar, which was

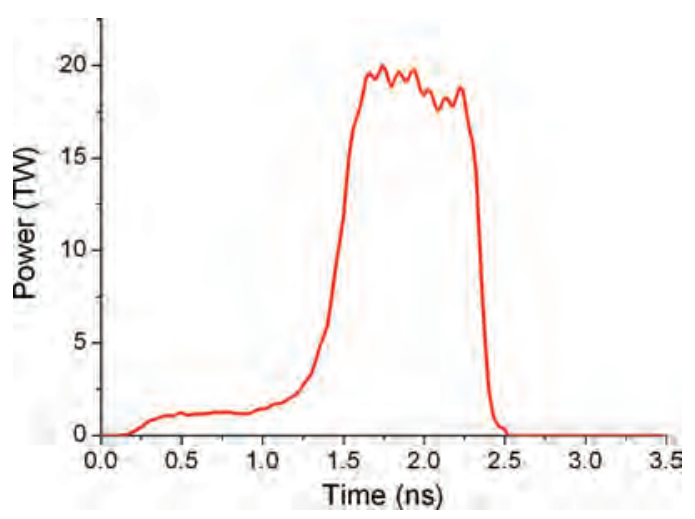

FIG. 1. (Color online) OMEGA laser pulse shape used in the experiment to drive a low-adiabatic implosion. added for diagnostic purposes, becomes highly ionized during the implosion, and its x-ray emissions were recorded by three identical MMI instruments fielded along three quasiorthogonal lines of sight. Each MMI consists of a pinhole array, multilayered Bragg mirror, and X-ray framing camera with microchannel plates (MCP) (Fig. 2). ${ }^{12-14}$ The pinhole array has about a thousand $10 \mu \mathrm{m}$ pinholes drilled on a tantalum substrate in a periodic hexagonal pattern, which, in turn, creates an array of implosion core images. The Bragg mirror consists of 300 bilayers ( $15 \AA$ thick each) of boron carbide and tungsten, and reflects the collection of core images yielding a spectral resolution $(E / \Delta E \sim 150)$ along the axis parallel to the plane of incidence. A gated x-ray framing camera equipped with MCP strips records the collection of core images onto a film. The three MMI instruments simultaneously recorded collections of gated, spectrally resolved implosion core images observed along three quasiorthogonal lines of sight using the OMEGA target chamber diagnostic ports labeled by TIM3 $(\theta=142.62, \phi=342.00)$, TIM4 $(\theta=63.44, \phi=342.00)$, and TIM5 $(\theta=100.81, \phi=$ 270.00) where the figures in the parentheses show the angular coordinates of the lines of sight in the spherical coordinate system. The angles between these diagnostic ports are $70.5^{\circ}$ between TIM3 and TIM5, 79.2 ${ }^{\circ}$ between TIM3 and TIM4, and $79.2^{\circ}$ between TIM4 and TIM5.

Figure 3(a) shows the raw MMI data recorded along TIM4. The raw MMI data consists of four frames, each of which was recorded at different times in the experiment. The top frame, frame 1 , is the earliest in time, and the bottom frame, frame 4 , is the latest. We observe that, as time goes by, intensity becomes stronger and also the size of the imploded core gets smaller. The voltage pulse sweeps across the MCP from left to right at a speed of $155 \mu \mathrm{m} / \mathrm{ps}$ thus, by taking into account the sweep speed the time difference between the left-most pixel column and the right-most pixel column is about 100 ps and the separation between nearby lines is smaller (e.g., $\Delta t \sim 10$ ps between argon He $\beta$ and Ly $\beta$ ). The time separation between adjacent frames is about 100 ps. The photon energy axis lies horizontally increasing from left to right, and one can observe the argon Ly $\alpha\left(1 s^{2} S\right.$ $\left.-2 p^{2} P\right)$, He $\beta\left(1 s^{2} S-1 s 3 p^{1} P\right)$, and Ly $\beta\left(1 s^{2} S-3 p^{2} P\right)$ emission line images on the lower energy range of the film, and titanium absorption lines close to the high-energy end of the spectral range.

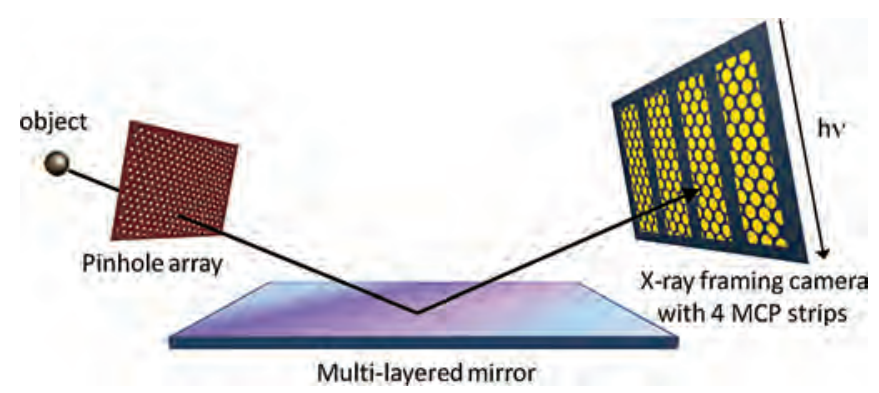

FIG. 2. (Color online) MMI consists of a pinhole array, multilayered Bragg mirror, and x-ray framing camera. The pinhole array creates a large number of object images, which are reflected off the mirror, and recorded by the four MCP strips of the framing camera. The dispersive effect of the mirror reflection results in an array of spectrally resolved images. 


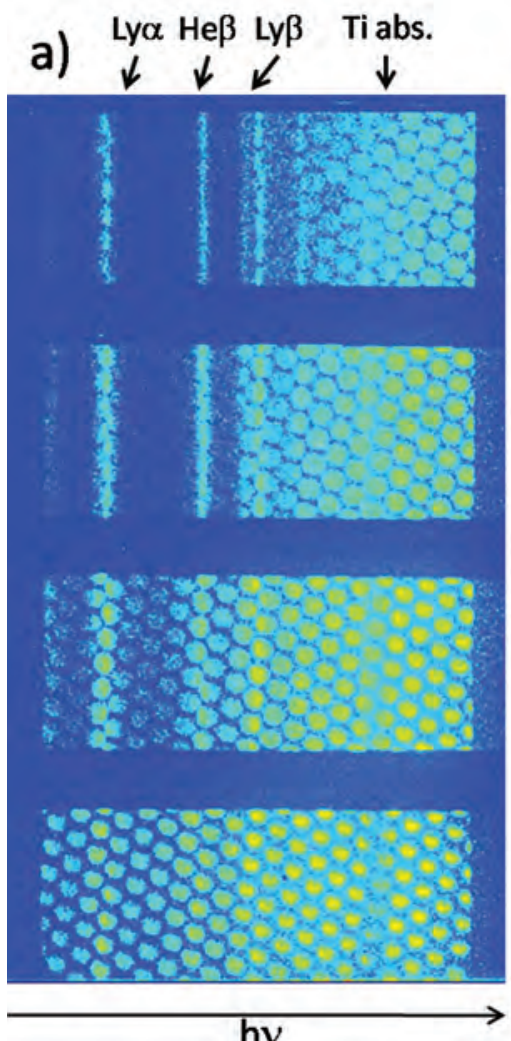

hv

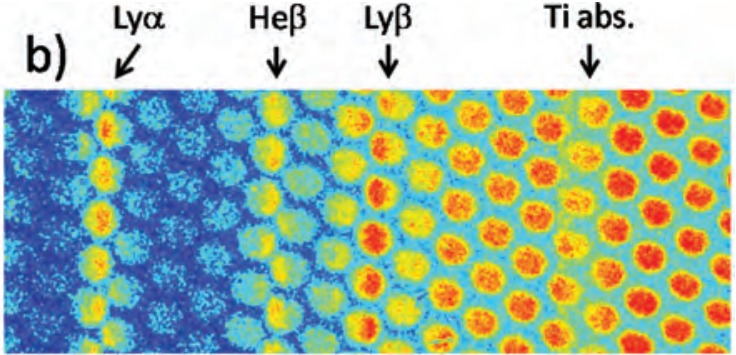

c)

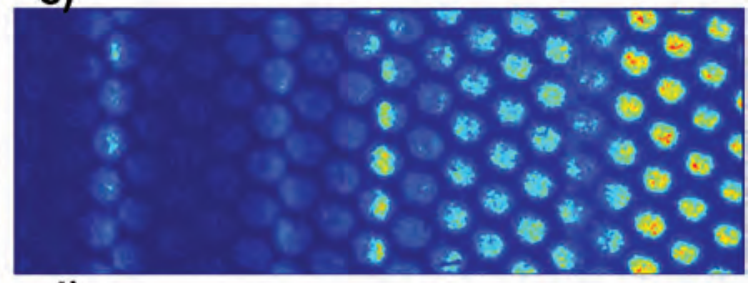

d)

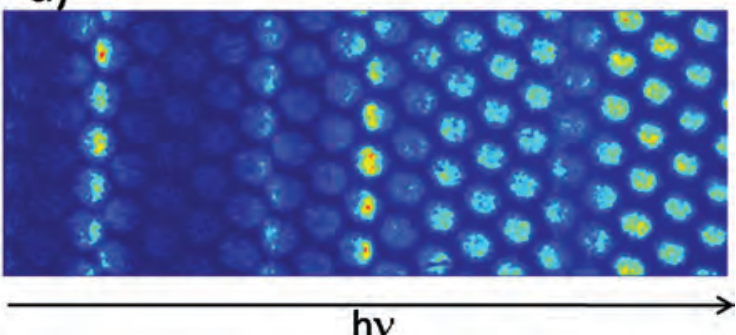

FIG. 3. (Color online) (a) Raw data recorded with TIM4, (b) frame 3 cropped from (a), (c) TIM4 frame 3 after wedge correction, (d) TIM4 frame 3 after photon-energy-dependent corrections.
Figure 3(b) shows the third frame of Fig. 3(a) (TIM4 frame3) before any corrections. The first correction to be applied is the conversion of film density to intensity using film calibration. Figure 3(c) shows the data after this correction. The next corrections are the photon-energy-dependent corrections associated with beryllium filter transmission, reflectivity of the multilayered Bragg mirror, and spectral response of the MCP. ${ }^{22}$ Figure 3(d) shows the data after these corrections. Once the film calibration, flat-fielding, and photon-energy-dependent corrections are applied, the centers of all the images are determined.

\section{IMAGE CENTER DETERMINATION}

In order to obtain broad- and narrowband images from the spectrally resolved image data, several core images have to be combined. This requires the use of a reference point for each image, which here we take as the center of the image.

The first method to determine the centers of the images on MMI data was developed by Welser et al. ${ }^{12}$ This method determined the centers based on the outer contour levels of each individual core subimages by assuming that periphery contour levels are dominated by continuum radiation contributions and thus less affected by spectral modulations due to line emissions. However, it is difficult to achieve accurate alignments between different core images as the centers are determined by individual contour levels. Also, the continuum level is not flat over these photon energy ranges. ${ }^{23,24}$ Thus, even if the contour levels are unperturbed by the argon line emissions, they could still be skewed by the continuum spectrum, which could lead to misalignment of the subi- mages. Alternatively, Izumi et al. introduced another algorithm to determine all the centers by fitting the Fourier transform of a parametrized two-dimensional wave pattern to that of MMI data, assuming that all pinholes and the resultant images are aligned with a periodic-parallel pattern consistent with pinhole array design. ${ }^{14}$ This algorithm finds the centers of all the core images simultaneously, and thus improves the alignments between different images. However, this technique could take a significant amount of computing time depending on the size of the data, because it repeatedly applies the FFT to the whole range of the image data. To process and analyze a large amount of data more efficiently, a faster center determination algorithm is preferred.

The new algorithm relies on the pattern of the dark regions in between images instead of the pattern of the core images. As the dark regions come purely from the pinhole array aperture and the location of the object, they are independent of object shape or spectral information. This new algorithm simultaneously determines all the centers by finding a parallel-line grid, which falls into the dark pattern in between the object images.

First, three adjacent subimages, which are not on the same straight line, are selected and their centers are, respectively, approximated based on intensity-weighted averages (see Fig. 4). These centers, $\mathbf{r}_{R}^{0}\left(x_{R}^{0}, y_{R}^{0}\right), \mathbf{r}_{C}^{0}\left(x_{C}^{0}, y_{C}^{0}\right)$, and $\mathbf{r}_{L}^{0}\left(x_{L}^{0}, y_{L}^{0}\right)$, provide the initial estimate of the parameters to define all the centers. A parallel-line grid that avoids all the centers is then constructed based on the origin, $\boldsymbol{O}$, as well as two basis vectors of the grid - namely, $\hat{\boldsymbol{u}}$ and $\hat{\boldsymbol{v}}$, which are defined as follows: 


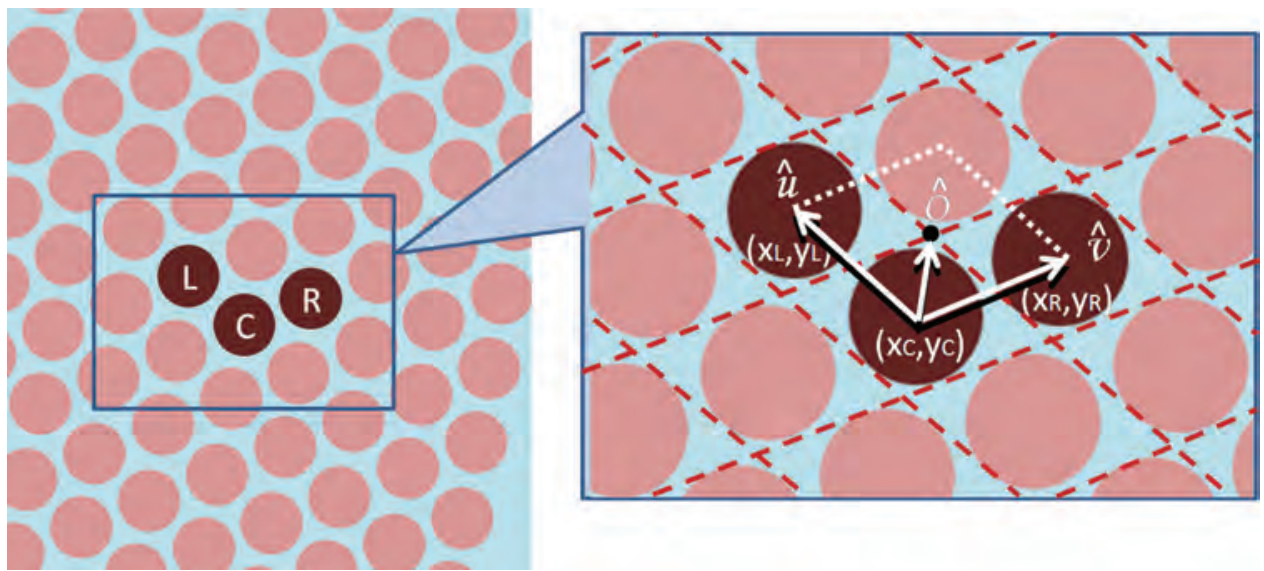

FIG. 4. (Color online) Three core images are selected, which are adjacent to each other but not on the same line. Then, based on their intensity-weightedaveraged centers, $\left(x_{C}, y_{C}\right),\left(x_{L}, y_{L}\right)$, and $\left(x_{R}, y_{R}\right)$, an initial estimate is made for the origin and two basis vectors of the parallel-line grid.

$$
\boldsymbol{O}=\boldsymbol{r}_{C}+\frac{\hat{\boldsymbol{u}}+\hat{\boldsymbol{v}}}{2}
$$

where $\hat{\boldsymbol{u}}=\boldsymbol{r}_{L}-\boldsymbol{r}_{C}$ and $\hat{\boldsymbol{v}}=\boldsymbol{r}_{R}-\boldsymbol{r}_{C}$. Based on the origin and the two basis vectors, parallel lines are iteratively constructed starting from the origin, and at the end, a parallelline grid is created as shown in Fig. 4. However, as one can observe in Fig. 5(a), these initially estimated grid lines go over some of the core subimages located far from the origin $\boldsymbol{O}$, as the parameters, $\boldsymbol{r}_{R}^{0}, \boldsymbol{r}_{C}^{0}, \boldsymbol{r}_{L}^{0}$ are not optimized yet. As the core images correspond to regions of high intensity of the data, the average intensity along this unoptimized parallelline grid is larger than that of the optimized one. Thus, Powells method ${ }^{25}$ is employed to find the optimal parameters $\boldsymbol{r}_{R}^{\text {opt }}, \boldsymbol{r}_{C}^{\mathrm{opt}}$, and $\boldsymbol{r}_{L}^{\mathrm{opt}}$ that minimize the average intensity along the resultant parallel grid. After this optimization, the resultant parallel lines fall in between all the core subimages, and the center of each diamond box is the center of each core image as in Fig. 5(b).

One important point is that this new center determination algorithm works in real space, instead of Fourier space, and thus provides better feedback to the operator and offers more direct control. Another point is that, as this new method
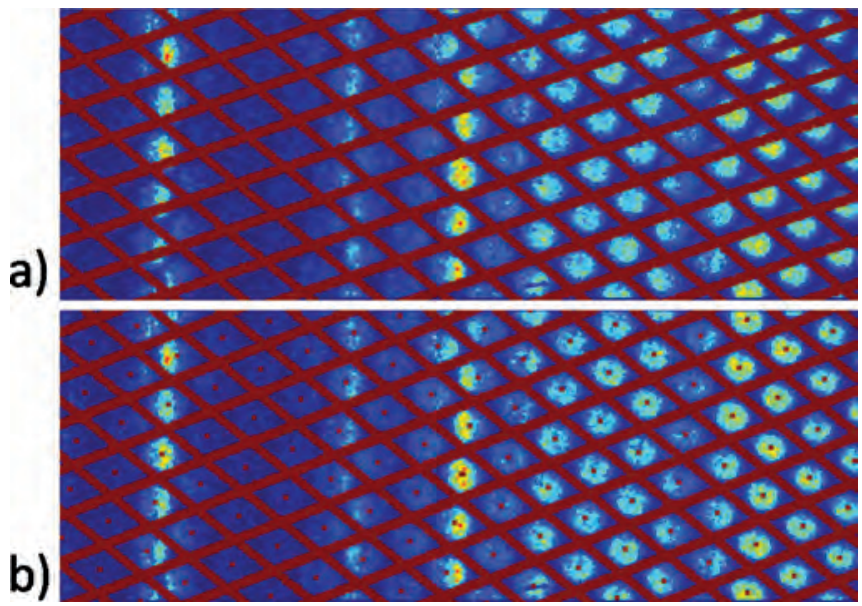

FIG. 5. (Color online) (a) Parallel line grid with the initial basis vectors of the grid. The grid does not avoid all the images. (b) After optimization of the basis vectors, the parallel-line grid matches the dark regions between images. The center of each diamond box is the center of each image. follows dark regions that suffer less from modulation of the signal, it provides very reliable and consistent results. To check the reliability of this technique, we repeated this center determination technique many times, each time starting with three different core images. Although different reference points, $\boldsymbol{O}$, were selected, the accuracy of the method can be accessed from the uncertainty of the two basis vectors. The mean values and the uncertainties of the basis vector components are expressed in polar coordinates as in $(d, \theta)$ : $\hat{\boldsymbol{u}}=\left(49.09 \pm 0.11\right.$ pixels, $\left.141.55^{\circ} \pm 0.05^{\circ}\right)$ and $\hat{\boldsymbol{v}}=(49.16 \pm$ 0.08 pixels, $21.24^{\circ} \pm 0.10^{\circ}$ ), which confirm the reliability of the method. For example, the uncertainty of $\hat{\boldsymbol{v}}$ shows that the possible misalignment between the center of one image to the center of its upper-right image (based on the definition of vector $v$ in Fig. 4) is 0.08 pixels. Then, the possible misalignment between the core image on lower left corner of Fig. 5 (b) to the one on the upper-right corner, which is the furthest away from the lower left image and separated approximately by 16 core images, is 0.08 pixels/image $\times 16$ images $\approx 1.3$ pixels, which corresponds to $3.0 \mu \mathrm{m}$ in the object space and much less than the spatial resolution of the instrument $(\sim 10 \mu \mathrm{m})$. Good center determination and alignment of the images are essential to extract narrowband or broadband images, and to estimate the shape and size of the implosion core.

\section{IMAGE RECONSTRUCTION}

Once all the centers are determined, one can reconstruct images of a given photon energy range (i.e., band). The reconstruction is based on the following equation: ${ }^{14}$

$$
i\left(x^{\prime}, y^{\prime}\right)=\frac{\sum_{j=0}^{N_{s}\left(x^{\prime}, y^{\prime}\right)-1} h\left(x^{\prime}+x_{j}, y^{\prime}+y_{j}\right)}{N_{s}\left(x^{\prime}, y^{\prime}\right)},
$$

where the two-dimensional arrays $i$ and $h$ correspond to the reconstructed core image and the MMI data, respectively and $\left(x_{j}, y_{j}\right)$ is the center position of the $j$ th core image on $h$, and $N_{s}\left(x^{\prime}, y^{\prime}\right)$ is the number of pixels contributing from the MMI data $h$. Figure 6(a) shows the MMI data denoted as $h(x, y)$ in Eq. (2). Figures 6(b)-6(d) show reconstructed images, denoted as $i\left(x^{\prime}, y^{\prime}\right)$ in Eq. (2), centered on argon $\operatorname{Ly} \beta$ over different energy ranges $\Delta E \approx 6,25$, and $68 \mathrm{eV}$, respectively. We note that if a very narrow band is selected, then the image 
(a) Ly $\alpha$

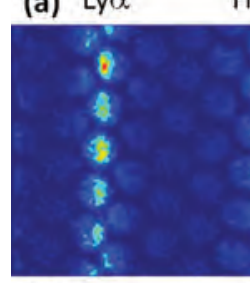

(b)

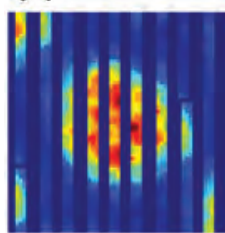

$\Delta \mathrm{E} \approx 6 \mathrm{eV}$
$\operatorname{He} \beta$ Ly $\beta$

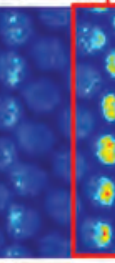

(c) $, 1,2,3,4,5,6$,

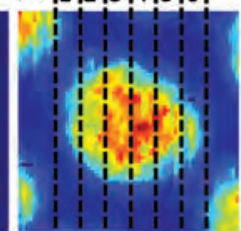

$\Delta \mathrm{E} \approx 25 \mathrm{eV}$

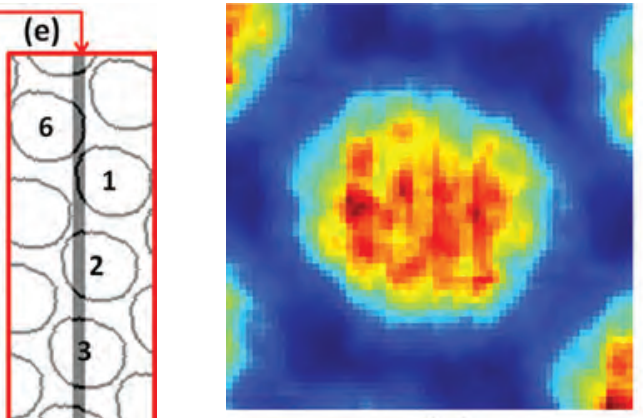

(a)

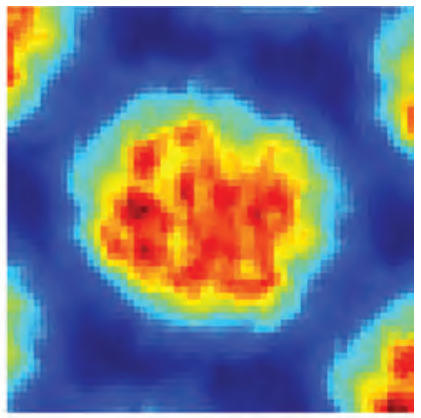

(b)

FIG. 7. (Color online) (a) Image without scaling and (b) image with intensity weighted reconstruction.

This artifact can be better avoided by separating the spectral content from the spatial content before reconstructing the image. This reconstruction method requires pieces of spatial information from a different spectral region within narrowband range. And, the intensity values depend not only on spatial region but also on photon energy. Thus, this vertical structure is created by the fact that the overall intensity drops significantly before filling up the whole image plane. Based on our theoretical analysis, we know that the relative spatial structure is rather insensitive to photon energy within the line profile, even though the overall intensity level itself changes significantly. Therefore, by scaling intensities properly before reconstructing the image, we can avoid this possible artifact. The spectral content can be removed by dividing each pixel value with the corresponding space-integrated intensity:

$$
h^{\prime}(x, y)=h(x, y) / \operatorname{spec}(x),
$$

where the two-dimensional array $h(x, y)$ and $h^{\prime}(x, y)$ represent the MMI data with and without spectral content, and $\operatorname{spec}(x)$ is the space-integrated spectrum discussed in Sec. V. Then, $h^{\prime}(x, y)$, instead of $h(x, y)$, is used in Eq. (2) to reconstruct the intensity weighted image. The reconstructed image is scaled back so that the final image has the same intensity level as the image reconstructed with the original technique, and also so that the reconstructed images from different lines have the appropriate intensity ratios. This rescaling is done as follows:

$$
i^{\prime}(x, y)=i(x, y) \frac{\sum_{n=x_{\min }}^{x_{\max }} \operatorname{spec}(x)}{x_{\max }-x_{\min }+1},
$$

where the two-dimensional array $i(x, y)$ and $i^{\prime}(x, y)$ represent before and after the final scaling, $x_{\min }$ and $x_{\max }$ represent the narrowband range for the image reconstruction in pixel number. Figure 7(b) shows the image using this intensity weighted reconstruction in the same color coding as Fig. 7(a). The vertical structure is removed as expected but shows the same intensity level.

We note that, as the core is imploding while the data are recoded over a finite time duration, there is potential for a blurring effect on the reconstructed images. However, the spatial extent of this effect is estimated to be $5 \mu \mathrm{m}$ and thus 
smaller than the $10 \mu \mathrm{m}$ spatial resolution of the instrument. Also, higher spatial resolution would be needed to observe the structure of spikes and bubbles characteristic of the mix region at the core-shell interface.

\section{SPACE-INTEGRATED SPECTRUM}

From the MMI data, one can extract not only images, but also a space-integrated spectrum. Basically, the spaceintegrated spectrum can be computed by summing up intensities vertically (i.e., perpendicular to the spectral dispersion axis) across the MMI data. Figure 8(a) shows the MMI data, and (b) the space-integrated spectrum by simply summing up all the intensities. Each vertical sum represents a space-integrated intensity of the corresponding photon energy on the spectral axis. However, this spectrum displays many artificial oscillations especially on the high-energy side, which is dominated by continuum emission and should be smooth. In this section, we introduce a correction technique to these artificial oscillations. Figure 8(c) shows the spectrum after the correction.
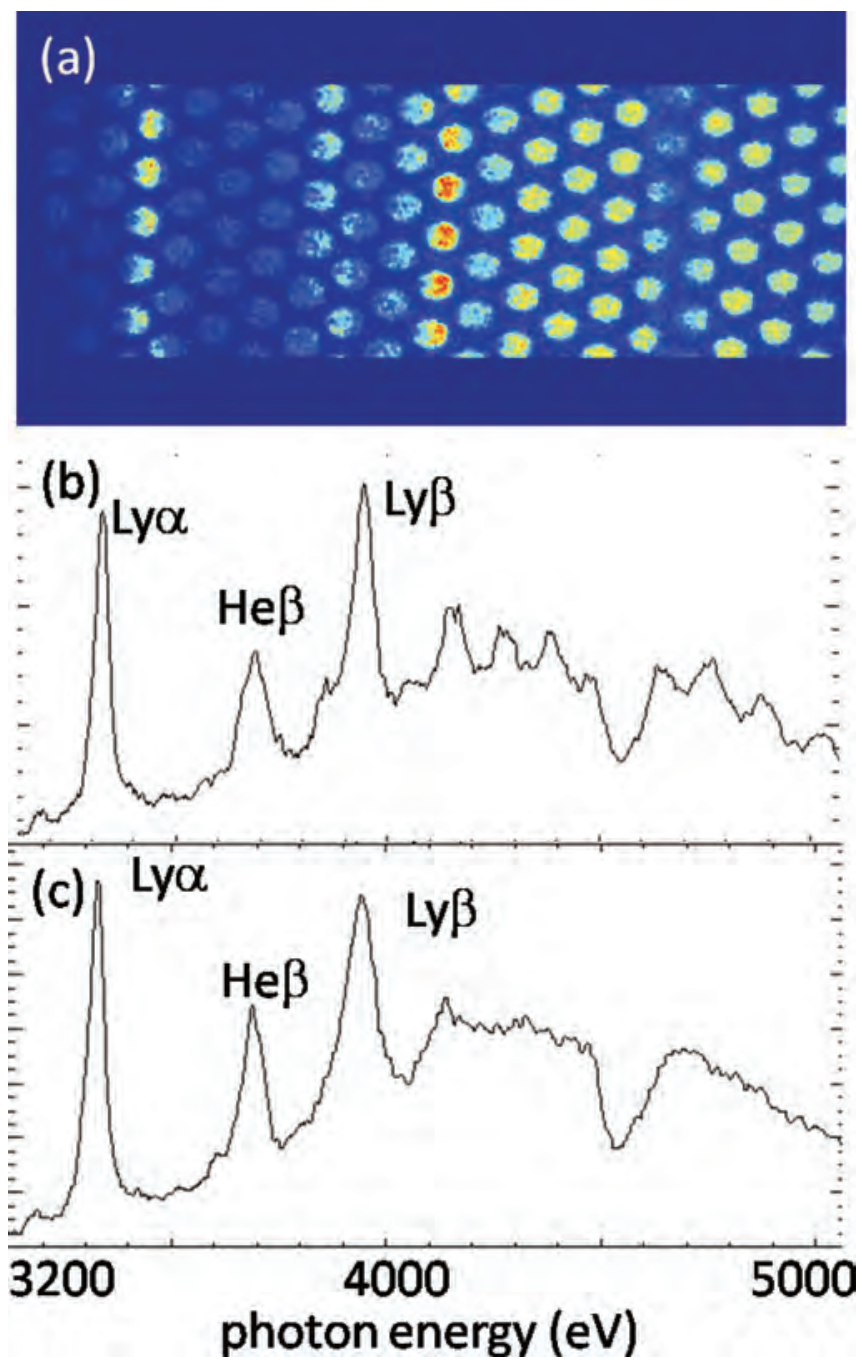

FIG. 8. (Color online) (a) MMI data after photon-energy-dependent corrections, (b) spectrum obtained by vertically summing up the intensities across (a); note the artificial oscillations, (c) spectrum with this artifact removed.
There are two major artifacts causing the oscillations shown in the MMI space-integrated spectrum. One artifact is due to the fact that the number of pixels contributing to the space-integrated spectrum for each photon energy is not constant, but changes periodically with respect to the $x$-axis (pixel or photon energy). Figure 9(a) shows the mask image of the MMI data where each circle represents the location and shape of the implosion core images. This mask is created by defining the core boundary image as the contour of the core broadband image and locating it at every center of the MMI core image data. Pixel values [Fig. 9(a)] are set to 1 if they are inside the core boundaries and set to 0 if outside. The spectrum of this mask represents the spectrum of the number of pixels contributing to the space-integrated spectrum as a function of horizontal pixel number, and implies that the MMI space-integrated spectrum oscillates partially due to differences in the number of contributing pixels. Another artifact is the bias in the spatial region representing space-integrated intensity. When the number of contributing pixels is small, those contributions tend to come from the periphery of the implosion core, which usually have lower

(a) Valley Peak
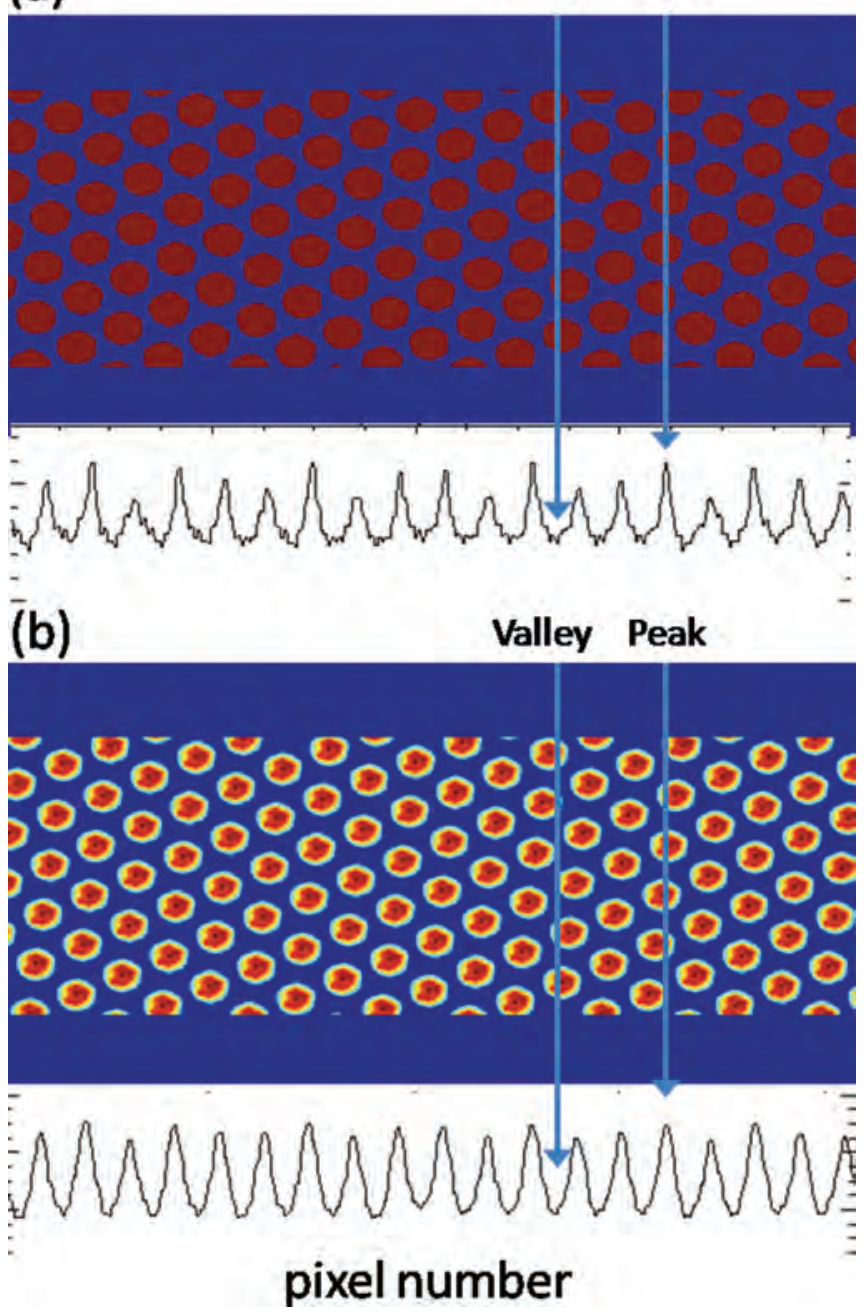

FIG. 9. (Color online) (a) Array of mask images and their spectrum; (b) array of broadband images and their spectrum, this spectrum is used to remove artificial oscillations from the space-integrated spectrum. 
intensity values as inferred by the lines on Fig. 9(a). To extract the oscillation spectrum taking into account both factors, we can replace each mask image in Fig. 9(a) with the broadband image as in Fig. 9(b). The resulting spectrum shows the oscillation taking into account both the difference in the number of contributing pixels and the bias in the spatial sampling points. By dividing the raw spectrum [Fig. 8(b)] by this oscillating spectrum, one can remove the artificial oscillations from the spectrum [Fig. 8(c)].

\section{CORE VOLUME SHAPE AND SIZE ESTIMATION}

The shape and size of the implosion core can be estimated by using three broadband images recorded along different lines of sight. Our data were recorded along three quasiorthogonal lines of sight. Thus, for the rest of the discussion, it is assumed that the number of lines of sight is three, but the idea is general and the same technique can be applied to data recorded along more than three lines of sight. In fact, the larger the number of lines of sight the more accurate the volume size and shape estimation. First, the observations along the lines of sight have to be simultaneous in time. This is accomplished by time correlating the time histories of characteristic emission lines (e.g. argon $\mathrm{He} \beta$ and $\mathrm{Ly} \beta$ ) of the four frames in the MMI gated data with the time-histories of the same line emission recorded with a streaked spectrometer. ${ }^{17}$ For the purpose of volume determination, "simultaneous" is defined as $\Delta t_{\max } \times v_{\text {imp }}<\Delta x_{\text {instrum, }}$, where $\Delta t_{\max }$ is the maximum time difference between data recorded along different lines of sight, $v_{\mathrm{imp}}$ is the shell implosion velocity, and $\Delta x_{\text {instrum }}$ is the spatial resolution of the instrument. For the particular shot that we have been discussing, we found that frame 3 of TIM3, TIM4, and TIM5 have times close enough to apply the volume determination algorithms discussed here.
We consider two different ways to determine the size and shape of the implosion core volume for frame 3 by utilizing the reconstructed broadband images for all the lines of sight simultaneously. Before looking into each method, we discuss the linear transformation connecting the global coordinate system defined in the OMEGA target chamber with the line-of-sight specific (local) coordinate labeled by $T$. The following line-of-sight specific linear transformation matrix takes into account the direction of the line of sight and orientation of the experimental images, and permits one to go back and forth between the global and $T$ (local) coordinates:

$$
\begin{gathered}
{\left[\begin{array}{c}
x_{T} \\
y_{T} \\
z_{T}
\end{array}\right]=R_{T}\left[\begin{array}{l}
x \\
y \\
z
\end{array}\right],} \\
R_{T}=R_{z}\left(\alpha_{T}-\beta_{T}\right) R_{y}\left(-\theta_{T}\right) R_{z}\left(-\phi_{T}\right),
\end{gathered}
$$

where $R_{y}$ and $R_{z}$, respectively, define counterclockwise rotations about the $y$ and $z$ axes as

$$
\begin{aligned}
& R_{y}(\gamma)=\left[\begin{array}{ccc}
\cos \gamma & 0 & \sin \gamma \\
0 & 1 & 0 \\
-\sin \gamma & 0 & \cos \gamma
\end{array}\right], \\
& R_{z}(\gamma)=\left[\begin{array}{ccc}
\cos \gamma & -\sin \gamma & 0 \\
\sin \gamma & \cos \gamma & 0 \\
0 & 0 & 1
\end{array}\right],
\end{aligned}
$$

and $R_{T}$ is the total rotation matrix for the line of sight, $T$, which is defined by angle $\theta_{T}$ and $\phi_{T}$ in the spherical coordinate system of the OMEGA target chamber. The rotation matrix, $R_{y}\left(-\theta_{T}\right) R_{z}\left(-\phi_{T}\right)$, rotates the whole system so that the line of sight, $T$, is aligned along the positive $z$-axis [see Figs. 10(a)-10(c)]. The final rotation matrix, $R_{z}\left(\alpha_{T}-\beta_{T}\right)$, corrects the orientation of the rotated system so that the

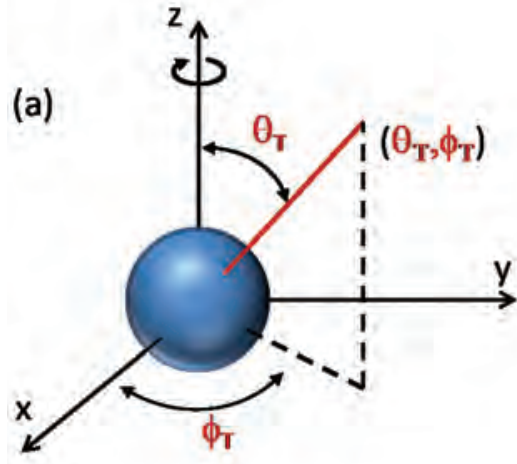

(d)

(c)

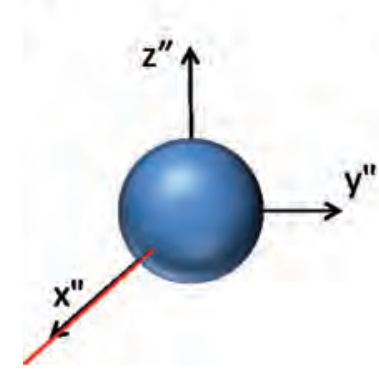

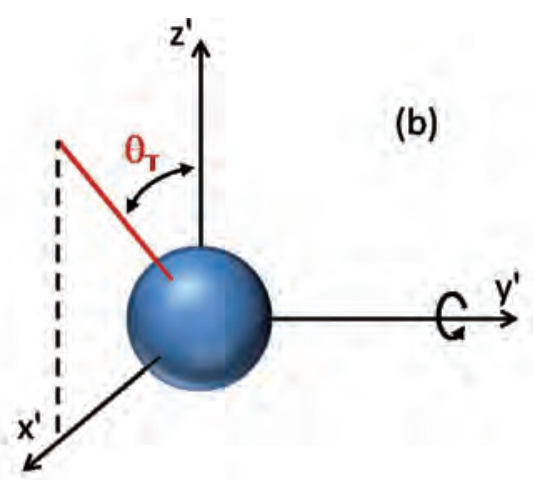

(e)
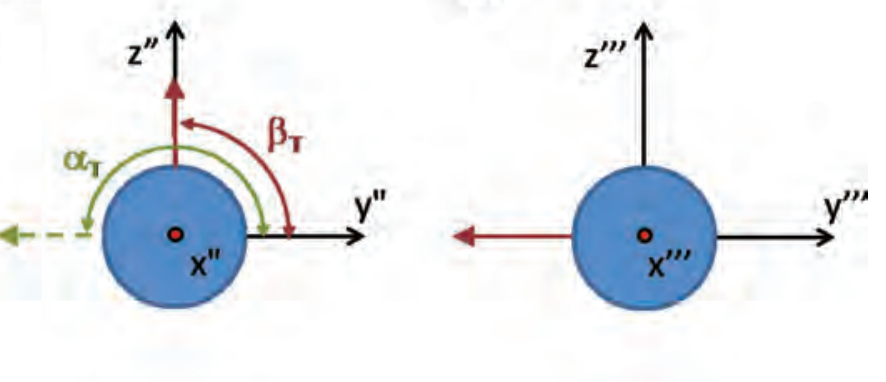

FIG. 10. (Color online) (a) $x-y-z$ coordinate defined in OMEGA target chamber and a line of sight defined by $\left(\theta_{T}, \phi_{T}\right)$, (b) after rotating $-\phi_{T}$ about the $z$-axis, (c) after rotating $-\theta_{T}$ about the $y$-axis, (d) this illustrates where the reference vector is seen on the $x^{\prime \prime}-y^{\prime \prime}$ plane and where it is supposed to be seen on the recorded image, (e) and after the final rotation to make the reference vector appear with the right orientation. 
projection of a reference vector in space appears at the proper angle on the image plane. In our case, and taking into account the image formation properties of the instrument, the projection of the $z$-axis defined in the chamber would appear at $180^{\circ}$ counterclockwise from the right of the image on the image plane, but it happens to appear at $180^{\circ}$ just after the rotation $R_{y}\left(-\theta_{T}\right) R_{z}\left(-\phi_{T}\right)$. The third rotation matrix is the identity $R_{z}\left(\alpha_{T}-\beta_{T}\right)=R_{z}(\pi-\pi)=I_{3}$. Hence, our procedure is able to correct the orientation using the third rotation matrix [Figs. 10(d) and 10(e)]. After applying the total rotation matrix, $R_{T}$, to the whole system, the line of sight, $T$, is aligned along positive $z$-axis, and by looking down along this axis toward the origin, one is supposed to see the implosion core in exactly the same shape and orientation as the extracted broadband image of this line of sight.

\section{A. Intersection of boundary projections}

The first method is based on the intersection of the projections of the core boundary images. For each line of sight, the boundary of the implosion core can be extracted by taking a contour line of the broadband image of the line of sight. An upper bound volume, size and shape, of the implosion core can be estimated by projecting those boundary images back in space along each line of sight and truncating an upper bound sphere volume by the intersection of those projections.

First, based on the maximum radius of the three boundary images, an upper bound sphere is defined in $x-y-z$ coordinates in the chamber. Then, one can reflect one boundary image on the volume by rotating the core volume with the appropriate rotation matrix, projecting the boundary image along this local $z$-axis, truncating the volume by this projection, and then undoing the rotation. By repeating this procedure for each of the three lines of sight, the shape and the size of the implosion core can be estimated. The size of the boundary images depends on what contour line is selected, thus an appropriate set of contour lines has to be used so that their projections do not overtruncate each other. The optimal contour lines are determined by a search and reconstruction method consisting of a Pareto genetic algorithm followed up by Levenberg-Marquardt least-squares minimization method. ${ }^{15}$ The Pareto genetic algorithm (PGA) is a multiobjective genetic algorithm that combines the Pareto domination technique of multiobjective optimization with the search and optimization genetic algorithm. The extracted volume is still an upper bound of the actual implosion core volume as not all shape details can be seen with only three lines of sight and some portions of the volume will still be defined by the upper bound sphere. More lines of sight observations would further improve the core volume size and shape estimation. Applying this technique to the implosion core volume at the time of frame 3 yields $4.4 \times 10^{-7} \mathrm{~cm}^{3}$. Based on the initial conditions of gas fill $\left(P_{D_{2}}=20 \mathrm{~atm}\right.$ at room temperature in a sphere of initial radius $400 \mu \mathrm{m}$ ), the total number of deuterium nuclei can be computed assuming an ideal gas equation of state; the result is $2.7 \times 10^{17}$. Then, based on the extracted upper bound core volume and assuming conservation of the deuterium mass, a lower bound for the deuterium mass density can be computed; this is $2.1 \mathrm{~g} / \mathrm{cm}^{3}$.

\section{B. Broadband images fitting}

The second method finds the three-dimensional "broadband emissivity" distribution in the core volume by fitting the broadband images observed along the three lines of sight, and uses the emissivity distribution to estimate the volume. ${ }^{26}$ To this end, the broadband emissivity distribution is written as a linear combination of Gaussian distribution functions as follows:

$$
\epsilon(x, y, z)=\sum_{i} A_{i} \exp \left[-\frac{\left(x-x_{i}\right)^{2}+\left(y-y_{i}\right)^{2}+\left(z-z_{i}\right)^{2}}{\sigma_{i}^{2}}\right],
$$

where $x, y$, and $z$ are the (global) coordinates defined in the OMEGA target chamber, $\epsilon(x, y, z)$ is the broadband emissivity in the core volume, and $A_{i}$ and $\sigma_{i}$ are free parameters to be determined for the Gaussian function centered at $\left(x_{i}, y_{i}, z_{i}\right)$. In this algorithm, the emissivity is assumed to be optically thin, and the relationship between experimental broadband images and the emissivity distribution can be written as

$$
P_{T}\left(x_{T}, y_{T}\right)=\int_{\text {rear }}^{\text {front }} \epsilon(x, y, z) d z_{T},
$$

where $x_{T}, y_{T}$, and $z_{T}$ are the local coordinates defined for each line of sight, $T, P_{T}\left(x_{T}, y_{T}\right)$ is the projection of the emissivity $\epsilon(x, y, z)$ along $z_{T}$ of the line of sight, which is equivalent to the formal integration of the radiation transport equation in the optically thin case. This assumption is appropriate as our broadband images are defined over argon $\mathrm{He} \beta$, $\mathrm{He} \gamma$, and Ly $\beta$ lines, which have small optical depths in argon-doped implosions. ${ }^{27,28}$

The goal of this method is to find the parameters $A_{i}$ and $\sigma_{i}$ for the 3D emissivity distribution, which can reproduce the experimental broadband images recorded along TIM3, TIM4, and TIM5. To search for the parameters we used a PGA followed up by a Levenberg-Marquardt (LM) minimization method to refine the parameters. ${ }^{15}$ The objectives of the PGA are the fits to each projection and a Laplacian physical constraint as defined in Ref. 29. This Laplacian constraint is introduced to reduce degenerate solutions by eliminating unphysical solutions with the assumption that the broadband emissivity distribution is a well-behaved and smooth function of position. The LM method is a singleobjective algorithm, thus all the objectives defined in the PGA have to be redefined into one objective as defined in Ref. 26. The new objective to be minimized is

$$
E_{\boldsymbol{A}, \boldsymbol{\sigma}}=\sum_{T}\left\|P_{\exp }^{T}-P_{\boldsymbol{A}, \boldsymbol{\sigma}}^{T}\right\|^{2}+\lambda\left\|L\left(\epsilon_{\boldsymbol{A}, \boldsymbol{\sigma}}\right)\right\|^{2}
$$

where the first summation term is the contribution from the fits, the second term is the Laplacian constraint, and $\lambda$ is a scaling constant to make the Laplacian constrain comparable to the other terms in the sum.

This is the first application of this method to MMI data recorded along three quasiorthogonal lines of sight. The result compares well with the shape and size of the implosion core found in Sec. VI A, as it is illustrated in Fig. 11. 


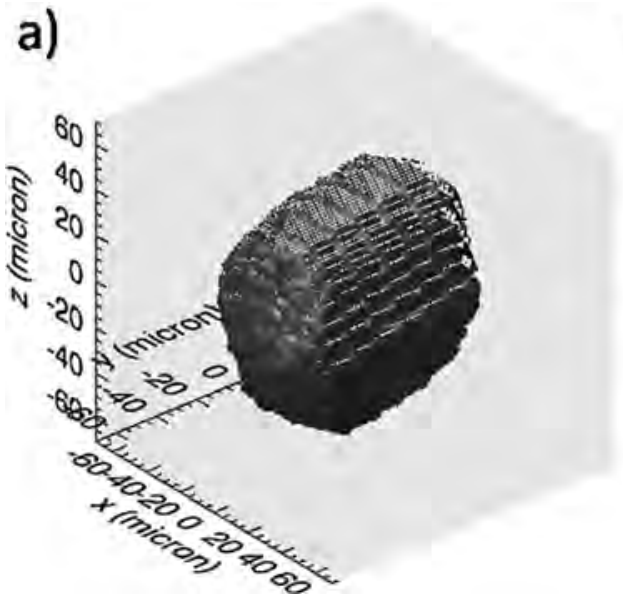

b)

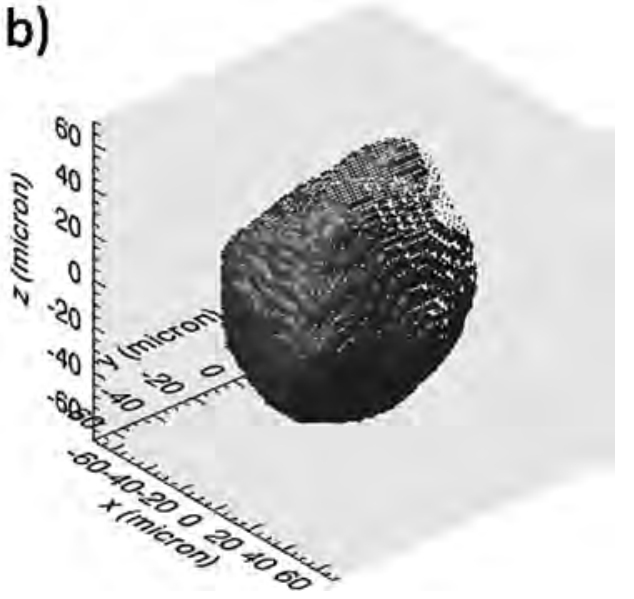

FIG. 11. Volume determination due to (a) intersection of boundary projections and (b) broadband images fitting. The two volumes compare well in both size and shape.

\section{SUMMARY AND CONCLUSIONS}

The MMI instrument records hundreds of gated, spectrally resolved $\mathrm{x}$-ray images of inertial confinement fusion implosion cores. The MMI data are rich in information and are resolved in photon energy, space, and time. To maximize the usefulness and diagnostic impact of the data, a good processing method is critical. ${ }^{12,14}$ This article summarizes advances and new developments in MMI data processing methods.

A method for finding all image centers simultaneously in the image array was discussed based on fitting a parallel-line grid to the pattern of dark regions in between the object images on the MMI data. This new center determination technique works in real space and it is very accurate and much faster for large data sets than the technique introduced by Izumi et al. ${ }^{14}$ We noted that artificial, vertical structures can be observed on reconstructed narrowband images. This artifact can be avoided with an intensity weighted image reconstruction, which takes into account the spectral intensity modulation as one fills the image plane. We also identified two sources of artifacts in the extraction of the space-integrated spectrum, and discussed how to remove them. Last, we discussed how to determine the shape and size of the implosion core volume based on two alternative algorithms using broadband images recorded along three-quasiorthogonal lines of sight. One method is based on the intersection of three boundary image projections, and the other one is based on the reconstruction of an optically thin emissivity distribution using a search and reconstruction method driven by a Pareto genetic algorithm. ${ }^{15}$ Both methods produced consistent results.

By processing and interpreting it properly, the gated MMI data have potential to unveil various important aspects of inertial confinement fusion experiments, such as changes in the implosion core volume shape and size, space-averaged changes in temperature and density based on space-integrated spectrum, ${ }^{27,28,30}$ and the evolution of electron temperature and density spatial structure based on the analysis of narrowband images and the space-integrated spectrum. $^{17}$

\section{ACKNOWLEDGMENTS}

This work was supported by DOE/NLUF Grant No. DEFG52-09NA29042 and by Lawrence Livermore National Laboratory under Contract No. DE-AC52-07NA27344.

${ }^{1}$ J. Nuckolls, L. Wood, A. Thiessen, and G. Zimmerman, Nature 239, 139 (1972).

${ }^{2}$ J. Lindl, Phys. Plasmas 2, 3933 (1995).

${ }^{3}$ S. Atzeni and J. Meyer-Ter-Vehn, The Physics of Inertial Fusion, edited by J. Birman, S. F. Edwards, R. Friend, M. Rees, D. Sherrington, and G. Veneziano (Clarendon, Oxford, 2004).

${ }^{4}$ S. Atzeni, Plasma Phys. Control. Fusion 51, 124029 (2009).

${ }^{5}$ M. Tabak, J. Hammer, M. E. Glinsky, W. L. Kruer, S. C. Wilks, and R. J. Mason, Phys. Plasmas 1, 1626 (1994).

${ }^{6}$ R. Betti, C. D. Zhou, K. S. Anderson, L. J. Perkins, W. Theobald, and A. A. Solodov, Phys. Rev. Lett. 98, 155001 (2007).

${ }^{7}$ J. A. Oertel, T. Archuleta, G. Peterson, and F. J. Marshall, Rev. Sci. Instrum. 68, 789 (1997).

${ }^{8}$ F. J. Marshall and J. A. Oertel, Rev. Sci. Instrum. 68, 735 (1997).

${ }^{9}$ Y. Ochi, I. Golovkin, R. Mancini, I. Uschmann, A. Sunahara, H. Nishimura, K. Fujita, S. Louis, M. Nakai, H. Shiraga, N. Miyanaga, H. Azechi, R. Butzbach, E. Forster, J. Delet- trez, J. Koch, R. W. Lee, and L. Klein, Rev. Sci. Instrum. 74, 1683 (2003).

${ }^{10}$ I. Uschmann, K. Fujita, I. Niki, R. Butzbach, H. Nishimura, J. Funakura, M. Nakai, E. Forster, and K. Mima, Appl. Opt. 39, 5865 (2000).

${ }^{11}$ K. Fujita, H. Nishimura, I. Niki, J. Funakura, I. Uschmann, R. Butzbach, E. Forster, M. Nakai, M. Fukao, A. Sunahara, H. Takabe, and T. Yamanaka, Rev. Sci. Instrum. 72, 744 (2001).

${ }^{12}$ L. A. Welser, R. C. Mancini, J. A. Koch, S. Dalhed, R. W. Lee, I. E. Golovkin, F. Marshall, J. Delettrez, and L. Klein, Rev. Sci. Instrum. 74, $1951(2003)$

${ }^{13}$ J. A. Koch, T. W. B. Jr., N. Izumi, R. Tommasini, R. C. Mancini, L. A. Welser, and F. J. Marshall, Rev. Sci. Instrum. 76, 073708 (2005).

${ }^{14}$ N. Izumi, T. W. Barbee, J. A. Koch, R. C. Mancini, and L. A. Welser, Rev. Sci. Instrum. 77, 083504 (2006).

${ }^{15}$ T. Nagayama, R. C. Mancini, L. A. Welser, S. Louis, I. E. Golovkin, R. Tommasini, J. A. Koch, N. Izumi, J. A. Delettrez, F. J. Marshall, S. P. Regan, V. A. Smalyuk, D. Haynes, and G. Kyrala, Rev. Sci. Instrum. 77, 10F525 (2006).

${ }^{16}$ R. Tommasini, J. A. Koch, N. Izumi, L. A. Welser, R. C. Mancini, J. Delettrez, S. Regan, and V. Smalyuk, Rev. Sci. Instrum. 77, 10E303 (2006).

${ }^{17}$ T. Nagayama, R. C. Mancini, R. Florido, R. Tommasini, J. A. Koch, J. A. Delettrez, S. P. Regan, V. A. Smalyuk, L. A. Welser-Sherrill, and I. E. Golovkin, Rev. Sci. Instrum. 79, 10E921 (2008).

${ }^{18}$ R. Tommasini, J. A. Koch, N. Izumi, L. A. Welser, R. C. Mancini, J. Delettrez, S. P. Regan, and V. Smalyuk, Proc. SPIE 6317, 31716 (2006).

${ }^{19}$ Y. Lin, T. J. Kessler, and G. N. Lawrence, Opt. Lett. 20, 764 (1995).

${ }^{20}$ K. Tsubakimoto, T. Jitsuno, N. Miyanaga, M. Nakatsuka, T. Kanabe, and S. Nakai, Opt. Commun. 103, 185 (1993).

${ }^{21}$ S. Skupsky, R. W. Short, T. Kessler, R. S. Craxton, S. Letzring, and J. M. Soures, J. Appl. Phys. 66, 3456 (1989). 
${ }^{22}$ G. A. Rochau, J. E. Bailey, G. A. Chandler, T. J. Nash, D. S. Nielsen, G. S. Dunham, O. F. Garcia, N. R. Joseph, J. W. Keister, M. J. Madlener, D. V. Morgan, K. J. Moy, and M. Wu, Rev. Sci. Instrum. 77, 10E323 (2006).

${ }^{23}$ B. Yaakobi, R. Epstein, and F. J. Marshall, Phys. Rev. A 44, 8429 (1991).

${ }^{24}$ F. J. Marshall, J. A. Delettrez, R. Epstein, and B. Yaakobi, Phys. Rev. E 49, 4381 (1994).

${ }^{25}$ W. H. Press, B. P. Flannery, S. A. Teukolsky, and W. T. Vetterling, Numerical Recipes in C (Cambridge University Press, Cambridge, 1997).

${ }^{26}$ Y.-W. Chen, T. Kohatsu, S. Nozaki, and R. Kodama, Rev. Sci. Instrum. 74, 2236 (2003).
${ }^{27}$ B. A. Hammel, C. J. Keane, D. R. Kania, J. D. Kilkenny, R. W. Lee, and N. D. Delamater, Rev. Sci. Instrum. 63, 5017 (1992).

${ }^{28}$ C. J. Keane, B. A. Hammel, D. R. Kania, J. D. Kilkenny, R. W. Lee, A. L. Osterheld, L. J. Suter, R. C. Mancini, J. C. F. Hooper, and N. D. Delamater, Phys. Fluids B 5, 3328 (1993).

${ }^{29}$ Y.-W. Chen, Z. Nakao, K. Arakaki, X. Fang, and S. Tamura, Fuzzy Sets Syst. 103, 285 (1999).

${ }^{30}$ R. Florido, R. C. Mancini, T. Nagayama, R. Tommasini, J. A. Delettrez, S. P. Regan, V. A. Smalyuk, R. Rodriguez, and J. M. Gil, High Energy Density Phys. 6, 70 (2010). 